\title{
PRODUCTS OF IDEMPOTENTS IN REGULAR RINGS
}

\author{
by K. C. O'MEARA
}

(Received 19 March, 1985)

1. Introduction. The problem of describing the subsemigroup generated by the idempotents in various natural semigroups has received the attention of several semigroup theorists ([1], [2], [3], [5], [7]). However, in those cases where the parent semigroup is in fact the multiplicative semigroup of a natural ring, the known ring structure has not been exploited. When this ring structure is taken into account, proofs can often be streamlined and can lead to more general arguments (such as not requiring that the elements of the semigroup be already transformations of some known structure).

In 1967, Erdos [3] showed that the linear transformations of a finite-dimensional vector space over a division ring which are products of proper $(\neq 1)$ idempotents are exactly the singular ones. Recently (in 1984), Reynolds and Sullivan [7] have obtained a corresponding characterization for linear transformations of an arbitrary vector space (of arbitrary dimension) over a division ring. (The author independently discovered this result in 1971 and referred to it, without proof, in [6, Remark on p. 19].) From the standpoint of Ring Theory, the parent semigroup involved here is the multiplicative semigroup of a full linear ring. As a ring, it is well known that a full linear ring can be characterized as a (von Neumann) regular, right self-injective ring which is prime and of Type I. Regular rings are well endowed with idempotents, and it has long been known that many of these rings are generated as rings by their idempotents (see [4, Theorem 13.16]), that is each element is a sum of products of idempotents. In this paper we complete the characterization of elements of a general prime, regular, right self-injective ring $Q$ (of Type I, II, or III) which can be written as a product of idempotents. Both the Erdos and the Reynolds and Sullivan results then follow as corollaries.

Our approach is very much ring-theoretic and does not require that the elements of $Q$ be mappings of some given structure. Instead we bring to bear standard results on regular rings, and supplement these with important facts about the ring structure of $Q$ that were discovered during 1973-76. In particular we use Goodearl's characterization of the ideals of $Q$, the Goodearl-Boyle infinite dimension function $\mu$ defined on the lattice of principal right ideals of $Q$, and the existence of a unique rank function on $Q$ (discovered by Goodearl and Handelman) when $Q$ is directly finite.

In the directly infinite case (Types $I_{\infty}, I_{\infty}, I I I$ ) our characterization of the elements of $Q$ which are expressible as products of idempotents (Theorem 6) closely resembles the Reynolds and Sullivan infinite-dimensional full linear ring result (Type $I_{\infty}$ ), but with the Goodearl-Boyle infinite dimension function $\mu$ taking over the role of vector space dimension. In the directly finite case (Types $\mathrm{I}_{\mathrm{f}}, \mathrm{II}_{\mathrm{f}}$ ), surprisingly the answer is the same for Type $\mathrm{II}_{\mathrm{f}}$ as Erdos' finite-dimensional full linear ring result (Type $\mathrm{I}_{\mathrm{f}}$ ): an element of $Q$ is a product of proper $(\neq 1)$ idempotents if and only if it is not a unit. We show in fact that this holds in any directly finite, simple, regular ring which satisfies the comparability axiom (Theorem 3).

Glasgow Math. J. 28 (1986) 143-152. 
2. Preliminaries. Rings considered in this paper are associative with an identity element. The unqualified term ideal always refers to a two-sided ideal. For a subset $X$ of a ring $R$, we let $r(X, R)=\{r \in R \mid X r=0\}$ denote the right annihilator of $X$ in $R$. Similarly $l(X, R)$ denotes the left annihilator. Modules are generally unital right modules. A module $A$ (over a general ring) is subisomorphic to a module $B$, written $A \leqslant B$, if $A$ is isomorphic to a submodule of $B$. For a module $A$ and cardinal $\alpha$, the direct sum of $\alpha$ copies of $A$ is denoted as $\alpha A$.

A ring $R$ is (von Neumann) regular if for each $x \in R$ there exists $y \in R$ such that $x y x=x$. If $y$ can be chosen to be a unit (i.e. invertible element) of $R$, then $R$ is unit-regular. The reader is referred to Goodearl [4] for properties of regular rings. With few exceptions, our notation and terminology follow [4]. A ring $R$ is right self-injective if the module $R_{R}$ is injective, that is for any right $R$-modules $A \subseteq B$ and $R$-homomorphism $f: A \rightarrow R$, there exists an extension $g: B \rightarrow R$ of $f$. The reader should consult Goodearl [4, Chapters 9 and 10] for the theory of regular, right self-injective rings and the associated theory of types. Such a ring is uniquely a direct product of rings of Types $\mathbf{I}_{\mathrm{f}}, \mathbf{I}_{\infty}, \mathbf{I I}_{\mathrm{f}}, \mathbf{I I}_{\infty}, \mathrm{III}$. If in addition a regular, right self-injective ring $R$ is prime $(a R b=0 \Rightarrow$ $a=0$ or $b=0$ ), or equivalently indecomposable, then $R$ must be exactly one of the above five types. In this case, for example, a Type $\mathrm{I}_{\mathrm{f}}$ prime $R$ is precisely a finite-dimensional full linear ring $\operatorname{End}_{D}(V)$ for a finite-dimensional right vector space $V$ over a division ring $D$, and a Type $\mathrm{I}_{\infty}$ prime $R$ is precisely an infinite-dimensional full linear ring. Representative examples of the other three types can be found in Goodearl [4, Chapter 10].

A module $A$ is directly finite if $A$ is not isomorphic to a proper direct summand of itself. Otherwise $A$ is directly infinite. A ring $R$ is directly finite if $x y=1$ implies $y x=1$; otherwise $R$ is directly infinite. (The ring $R$ being directly finite is equivalent to the module $R_{R}$ being directly finite.) A regular ring $R$ is said to satisfy the comparability axiom if, for any $x, y \in R$, either $x R \leqslant y R$ or $y R \leqslant x R$.

A rank function on a regular ring $R$ is a map $N: R \rightarrow[0,1]$ which satisfies:

(a) $N(1)=1$,

(b) $N(x y) \leqslant N(x)$ and $N(x y) \leqslant N(y)$ for all $x, y \in R$,

(c) $N(e+f)=N(e)+N(f)$ for all orthogonal idempotents $e, f \in R$,

(d) $N(x)>0$ for all nonzero $x \in R$.

Goodearl and Handelman (1975) established, for example, that every directly finite, simple, regular ring which satisfies the comparability axiom has a (unique) rank function [4, Corollary 16.15].

Let $R$ be a prime, regular, right self-injective ring. The Goodearl-Boyle (1976) infinite dimension function $\mu$, defined on the class of nonsingular injective right $R$-modules, is given by $\mu(A)=0$ if $A=0$, while if $A \neq 0$ then

$\mu(A)$ is the smallest infinite cardinal $\alpha$ such that $\alpha A \$ A$.

(See [4, Chapter 12], where $\mu$ is the same as the function $\mu_{M}$ for $M=\{0\}$.) For example, if $R$ is a field and $A$ is an infinite-dimensional vector space over $R$, then $\mu(A)$ is the successor of $\operatorname{dim} A$. The function $\mu$ satisfies the two basic properties one expects of a 
"dimension function", namely

$$
\begin{aligned}
& A \leqslant B \Rightarrow \mu(A) \leqslant \mu(B), \\
& \mu(A \oplus B)=\mu(A)+\mu(B) .
\end{aligned}
$$

See [4, Proposition 12.6]. If $A$ and $B$ are directly infinite, then conversely $\mu(A) \leqslant \mu(B)$ implies $A \leqslant B$ [4, Corollary 12.11], and consequently $\mu$ actually determines the isomorphism classes of the directly infinite, nonsingular, injective modules. We are mostly interested in $\mu(x R)$ for principal right ideals $x R$ of $R$. In this setting it is convenient for the statement of our main result to define the $\mu$-codimension of $x R$ to be

$$
\mu \text { - } \operatorname{codim}(x R)=\mu(y R)
$$

for any complement $y R$ of $x R$ (that is $R=x R \oplus y R$ ). Notice that $\mu(x R)=\aleph_{0}$ for any nonzero directly finite $x R$, so $\mu(x R)$ gives no information in this case apart from saying $x R$ is nonzero and directly finite. (For this situation one can turn to the relative dimension functions in [4, Chapter 11] or use a rank function on a suitable subring of $R$ containing $x$.)

In terms of $\mu$, Goodearl's (1973) characterization of the ideals of a prime, regular, right self-injective ring $R$ is that they are precisely of the form

$$
H(\alpha)=\{x \in R \mid \mu(x R) \leqslant \alpha\}
$$

for infinite cardinals $\alpha$ [4, Proposition 12.19]. In particular the ideals are well-ordered under inclusion. Notice that $H\left(\aleph_{0}\right)$ is the ideal consisting of all $x \in R$ for which the module $x R$ is directly finite, and if $H\left(\aleph_{0}\right) \neq 0$, then $H\left(\aleph_{0}\right)$ is the unique minimum ideal of $R$. For a full linear ring $R$ (i.e. $R$ of Type I), the ideal $H\left(\aleph_{0}\right)$ consists of the linear transformations of finite rank.

Proposition 1. For elements $a, b$ of a prime, regular, right self-injective ring $R$, if $\mu(a R)=\mu(b R)$ then $R a R=R b R$.

Proof. This is immediate from the above description of the ideals of $R$.

We conclude our preliminaries with a simple but useful result on products of idempotents.

Proposition 2. Suppose $e$ is an idempotent of a regular ring $R$ such that $e R \leqslant(1-e) R$. Then every element $x \in e R e$ is expressible as a product of idempotents of $R$. Now

Proof. Since $e R \leqslant(1-e) R$, there exist $y \in e R(1-e)$ and $z \in(1-e) R e$ with $y z=e$.

$$
x=[e+x y][1-e][e+z]
$$

with all bracketed terms idempotent.

Note. (1) For an idempotent $e$ and element $b \in e R(1-e)$ or $b \in(1-e) R e$, the element $e+b$ is idempotent. We use this observation frequently. 
(2) Suppose $R$ is a regular ring and $a \in R$. If $a=a b a$ for $b \in R$, then $a R=e R$ and $R a=R f$, where $e=a b$ and $f=b a$ are idempotents and $e R \cong f R$. Also for any pair of idempotents $e, f \in R$, we have $e R \cong f R$ (as right $R$-modules) if and only if $e=y z$ and $f=z y$ for some $y \in e R f, z \in f R e$.

3. The directly finite case (Types $\left.I_{f}, I_{f}\right)$. We shall deduce the characterization of elements which are products of idempotents in a directly finite, prime, regular, right self-injective ring from the following natural extension of Erdos' result [3].

THEOREM 3. Let $R$ be a directly finite, simple, regular ring which satisfies the comparability axiom. Let $a \in R, a \neq 1$. Then $a$ is a product of idempotents if and only if $a$ is not a unit.

Proof. By [4, Corollary 16.15], $R$ has a unique rank function $N$. We prove by induction on $n$ that for all positive integers $n$ (and for all $R$ ):

$$
N(a) \leqslant(n-1) / n \Rightarrow a \text { is a product of idempotents. }
$$

This certainly holds for $n=1$ because then $a=0$. Assume the result for some $n$ (and for all $R$ ), and suppose $N(a) \leqslant n /(n+1)$. Clearly we can suppose $a \neq 0$. Let $r=N(a)$. Let $a R=e R$ for some $e=e^{2} \in R$. Note $N(e)=N(a)=r$. Let $A=e R e, b=a e, c=a(1-e)$. Let $f$ and $g$ be idempotents of $A$ such that

$$
b A=f A, \quad A b=A g .
$$

Since a directly finite, regular ring satisfying the comparability axiom is unit-regular $[4$, Theorem 8.12], $R$ is unit-regular and hence so also is $A$ [4, Corollary 4.7]. From $g A \cong f A$ we conclude by [4, Theorem 4.5] that $(e-g) A \cong(e-f) A$. Hence there exists $d \in(e-$ $f) A(e-g)$ such that $b+d$ is a unit of $A$. Let $u=b+d \in A$ and let $v \in A$ be its inverse. We have

$$
\begin{aligned}
a=b+c & =((b+d)+c)(g+(1-e)) \\
& =u[e+v c][g+(1-e)]
\end{aligned}
$$

with the two square-bracketed terms being idempotents. It suffices therefore to show that any $u \in A$ is a product of idempotents of $R$.

If $e R \leqslant(1-e) R$, then $u$ is a product of idempotents by Proposition 2 and we are finished. Since $R$ satisfies the comparability axiom, we may therefore assume that $(1-e) R \leqslant e R$. Write

$$
e=e_{1}+e_{2} \text {, }
$$

where $e_{1}, e_{2}$ are orthogonal idempotents of $A$ such that $e_{2} R \cong(1-e) R$. Choose $w \in e_{2} R(1-e), x \in(1-e) R e_{2}$ such that $w x=e_{2}$. Let $a_{1}=u e_{1} \in A$. Observe that $A$ is also a directly finite, simple, regular ring which satisfies the comparability axiom $[4$, Corollary 8.3]. Let $N_{1}$ be its unique rank function. Since $e=e_{1}+e_{2}$ with $N\left(e_{2}\right)=N(1-e)=$ $1-N(e)=1-r$, we have $N\left(e_{1}\right)=N(e)-N\left(e_{2}\right)=r-(1-r)=2 r-1$. Hence (see [4, 
Lemma 16.2])

$$
N_{1}\left(e_{1}\right)=N\left(e_{1}\right) / N(e)=(2 r-1) / r .
$$

Now $r \leqslant n /(n+1)$ implies $(2 r-1) / r \leqslant(n-1) / n$, whence $N_{1}\left(a_{1}\right) \leqslant N_{1}\left(e_{1}\right)=(2 r-1) / r \leqslant$ $(n-1) / n$. Thus

$$
N_{1}\left(a_{1}\right) \leqslant(n-1) / n \text {. }
$$

We can now apply the induction hypothesis to $a_{1}$ as a member of the ring $A=e R e$ and obtain

$$
a_{1}=f_{1} f_{2} \ldots f_{m}
$$

for some idempotents $f_{i} \in e R e$. Then

$$
a_{1}+(1-e)=\left[f_{1}+(1-e)\right]\left[f_{2}+(1-e)\right] \ldots\left[f_{m}+(1-e)\right]
$$

is a product of idempotents. Since

$$
u=[e+u w]\left[a_{1}+(1-e)\right][e+x]
$$

with the first and last square-bracketed terms idempotent, we have $u$ as a product of idempotents. Thus the induction works.

If $a$ is not a unit of $R$, then $N(a)<1$ and hence $N(a) \leqslant(n-1) / n$ for some $n$. Then $a$ is a product of idempotents. Conversely, if $a$ is a product of idempotents, then $a$ is clearly not a unit because $a \neq 1$.

Corollary 4. Let $Q$ be a directly finite, prime, regular, right self-injective ring. Let $a \in Q, a \neq 1$. Then $a$ is a product of idempotents if and only if $a$ is not a unit.

Proof. $Q$ satisfies the comparability axiom by [4, Corollary 9.16], and is simple by [4, Corollary 9.26].

Corollary 5 (Erdos [3], Dawlings [1]). For a division ring $D$ and positive integer $n$, an element $a \in M_{n}(D)$ is a product of proper $(\neq 1)$ idempotents if and only if $a$ is a singular matrix.

Proof. $M_{n}(D)$ is a directly finite, prime, regular, right self-injective ring (of Type $\mathrm{I}_{\mathrm{f}}$ ).

4. The general case. Our principal result is the following theorem.

THEOREM 6. Let $Q$ be a prime, regular, right self-injective ring. Let $a \in Q, a \neq 1$. Then $a$ is a product of idempotents if and only if either

or

(i) $\mu(r(a, Q))=\mu$-codim $(a Q)=\mu((1-a) Q)>\aleph_{0}$

(ii) a has the form $1+x, x \in H\left(\aleph_{0}\right)$, a not a unit of $Q$.

Remark. Condition (ii) is equivalent to

$$
0<\mu(r(a, Q))=\mu-\operatorname{codim}(a Q)=\mu((1-a) Q)=\aleph_{0},
$$

but this latter condition is not particularly illuminating because of the definition of $\mu(A)$ 
when $A$ is directly finite. However it does enable us to replace the two conditions by the single condition

$$
0<\mu(r(a, Q))=\mu-\operatorname{codim}(a Q)=\mu((1-a) Q) .
$$

In preparation for the proof of Theorem 6 we begin with some lemmas. Throughout this section $Q$ denotes a prime, regular, right self-injective ring. Let $M$ be the (unique) maximal ideal of $Q$, and for $x \in Q$ let $\bar{x}=x+M \in Q / M$. Note by [4, Corollary 2.23] that when $Q$ is directly infinite, $\bar{x} \neq 0$ if and only if $x Q \cong Q$.

Lemma 7. Let $e$ and $f$ be idempotents of $Q$. If $\overline{(1-f) Q} \nsubseteq \overline{e Q}$, then there exists an idempotent $g$ of $Q$ with $e Q f \subseteq g Q g$ and $\overline{1-g} \neq \overline{0}$.

Proof. Write $(1-f) Q=((1-f) Q \cap e Q) \oplus f_{1} Q$ and

$$
(1-e) Q=((1-e) Q \cap[(1-f) Q+e Q]) \oplus h Q
$$

for some $f_{1}, h \in Q$. Then

$$
\begin{aligned}
Q & =e Q+(1-e) Q \\
& =e Q \oplus h Q \oplus f_{1} Q .
\end{aligned}
$$

Let $g=g^{2} \in Q$ be such that $g Q=e Q \oplus h Q$ and $(1-g) Q=f_{1} Q$. We then have $e Q \subseteq g Q$ and $(1-g) Q \subseteq(1-f) Q$, whence $e Q f \subseteq g Q g$. If $\overline{1-g=\overline{0}}$ then $\overline{f_{1} Q}=\overline{0}$ and $\overline{(1-f) Q}=$ $\overline{(1-f) Q \cap e Q}$, which implies $\overline{(1-f) Q \subseteq e Q}$, a contradiction. Hence $\overline{1-g} \neq \overline{0}$.

Lemma 8. Let $J$ be a (two-sided) ideal of $Q$. For any $x \in J$, there exists an idempotent $g \in J$ with $x \in g Q g$.

Proof. Let $e$ and $f$ be idempotents of $Q$ such that $x Q=e Q$ and $Q x=Q f$. Let $g=g^{2} \in Q$ be constructed exactly as in the previous lemma, so that $g Q=e Q \oplus h Q$. Then $h Q \cap(1-f) Q=0$ implies $h Q \leqslant f Q \subseteq J$, which in turn implies $h \in J$ because $Q$ is regular (see Lemma 10(i)). Now $g Q=e Q+h Q \subseteq J$, giving $g \in J$. Also $x \in e Q f \subseteq g Q g$.

Lemma 9. Let $a \in Q$. Let $K=r(a, Q)$ and let $C$ be a complement right ideal of $a Q$ (relative to $Q$ ). Suppose

$$
Q K=Q \text { and } Q C=Q \text {. }
$$

If $Q$ is directly infinite, then $a$ is a product of idempotents.

Proof. Assume $Q$ is directly infinite. Let $e$ and $f$ be idempotents of $Q$ such that $a Q=e Q$ and $Q a=Q f$. Then our assumption (*) amounts to $\overline{1-f} \neq \overline{0}$ and $\overline{1-e} \neq \overline{0}$. Let $b=a e$ and $c=a(1-e)$. We consider two cases.

Case 1. Suppose $Q e=Q b \oplus Q h$ for some $h$ with $\bar{h}=\overline{0}$. Then $\overline{Q e}=\overline{Q b}=\overline{Q a e}=\bar{Q} \bar{f} \bar{e}$ implies $r(\bar{f}, \bar{e} \bar{Q})=\overline{0}$ and hence $\overline{(1-f) Q} \notin \bar{e} \bar{Q}$ (because $\overline{1-f} \neq \overline{0}$ ). By Lemma 7 , we have $a \in e Q f \subseteq g Q g$ for some $g=g^{2} \in Q$ with $\overline{1-g} \neq \overline{0}$. Since $Q$ is directly infinite and $\overline{1-g} \neq \overline{0}$, we have $(1-g) Q \cong Q$ and hence $g Q \leqslant(1-g) Q$. Proposition 2 now implies $a$ is a product of idempotents because $a \in g Q g$.

Case 2. Suppose $Q e=Q b \oplus Q h$ with $\bar{h} \neq \overline{0}$. We can assume $e=g+h$, where $g$ and $h$ are orthogonal idempotents in $e Q e$ and $Q b=Q g$. Since $Q$ is directly infinite and $\bar{h} \neq \overline{0}$, 
we have $h Q \cong Q$ and hence $Q=u Q$ for some $u \in Q h$. We can consequently find $v \in e Q h$ and $w \in h Q$ such that $e=v w$. Now

$$
a=b+c=(b+v)[e+w c][g+(1-e)] .
$$

The square-bracketed terms are idempotents, while $b+v \in e Q e$ is a product of idempotents by Proposition 2, since $\overline{1-e} \neq \overline{0}$ implies $e Q \leqslant(1-e) Q$.

Lemma 10. Let $R$ be any regular ring and let $J$ be an ideal of $R$. Let $a \in R$.

(i) For $x \in R$, if $x R \leqslant J$ then $x \in J$.

(ii) If $r(a, R) \subseteq J$, then $r(a+J, R / J)=0$.

(iii) If $J$ contains a complement of aR (relative to $R)$, then $l(a+J, R / J)=0$.

(iv) If $a$ is a product of idempotents and $r(a, R) \subseteq J$, then $1-a \in J$.

(v) If $a$ is a product of idempotents and $J$ contains a complement of $a R$, then $1-a \in J$.

Proof. (i) Suppose there is an isomorphism $\eta: y R \rightarrow x R$ for some $y \in J$. Since $R$ is regular, $\eta$ is given by left multiplication by an element of $R$, and so $x R=\eta y R \subseteq R y R \subseteq J$. Thus $x \in J$.

(ii) Assume $r(a, R) \subseteq J$. Let $b \in R$ and suppose $a b \in J$. Write $b R=(r(a, R) \cap b R) \oplus$ $c R$ for some $c \in R$. By (i), $c R \cong a c R \subseteq a b R \subseteq J$ implies $c \in J$. Hence, since $r(a, R) \subseteq J$, we have $b \in J$.

(iii) Assume $J$ contains a complement $c R$ of $a R$. Let $b \in R$ and suppose $b a \in J$. Write $a R=(a R \cap r(b, R)) \oplus x R$ for some $x \in R$. Since $x R \cong b x R \subseteq b a R \subseteq J$, we have $x \in J$ by (i). Also $R=(a R \cap r(b, R))+x R+c R$ and so $b R=b(x R+c R)$, whence by the projectivity of $b R$ we obtain $b R \leqslant x R+c R \subseteq J$. Another application of (i) now gives $b \in J$.

(iv) Suppose $a=e_{1} e_{2} \ldots e_{n}$ for some idempotents $e_{i} \in R$. Assume $r(a, R) \subseteq J$. For each $y \in R$, let $\bar{y}=y+J \in R / J$. Now $\bar{a}=\bar{e}_{1} \bar{e}_{2} \ldots \bar{e}_{n}$ and therefore, since $r(\bar{a}, \bar{R})=0$ by (ii), we must have each $\bar{e}_{i}=\overline{1}$. Hence $\bar{a}=\overline{1}$ and $1-a \in J$.

(v) This follows from an entirely similar argument using (iii).

We are now ready to prove the theorem.

Proof of Theorem 6. Suppose $a$ is a product of idempotents. Notice that always $r(a, Q) \subseteq(1-a) Q$ and so $\mu(r(a, Q)) \leqslant \mu((1-a) Q)$. Also if we write $(1-a) Q=$ $((1-a) Q \cap a Q) \oplus b Q$ for some $b \in Q$, then $b Q$ is a complement of $a Q$ contained in $(1-a) Q$, whence $\mu$-codim $(a Q) \leqslant \mu((1-a) Q)$.

Since $r(a, Q)$ is contained in the ideal $J=\{y \in Q \mid \mu(y Q) \leqslant \mu(r(a, Q))\}$, we have $1-a \in J$ by Lemma 10 (iv). Hence $\mu((1-a) Q) \leqslant \mu(r(a, Q))$, giving $\mu(r(a, Q))=$ $\mu((1-a) Q)$. (Note that essentially the argument shows $Q(1-a) Q=\operatorname{Qr}(a, Q)$ by Lemma 10(iv) and then uses the converse of Proposition 1.) Similarly the complement $b Q$ lies in the ideal $I=\{y \in Q \mid \mu(y Q) \leqslant \mu(b Q)\}$ and so by Lemma $10(v)$ we have $1-a \in I$. Hence $\mu((1-a) Q) \leqslant \mu-\operatorname{codim}(a Q)$, giving $\mu((1-a) Q)=\mu-\operatorname{codim}(a Q)$.

If $\mu(1-a) Q)>\aleph_{0}$, we now have (i). Suppose $\mu((1-a) Q) \leqslant \aleph_{0}$. Then $a-1 \in H\left(\aleph_{0}\right)$ and $a=1+(a-1)$ has the form (ii), since the only unit which is a product of idempotents is 1 .

For the converse, firstly assume (i). Let $\alpha=\mu((1-a) Q)$ and let $J=H(\alpha)$. Let 
$x=a-1$. Since $x \in J$, by Lemma 8 we can find $g=g^{2} \in J$ such that $x \in g Q g$. Let $y=g+x \in g Q g$. Note that $\alpha=\mu(x Q) \leqslant \mu(g Q) \leqslant \alpha$, whence $\mu(g Q)=\alpha$.

We claim: $y$ satisfies the condition $\left({ }^{*}\right)$ of Lemma 9 relative to the ring $g Q g$.

To verify this, let $A=g Q g, K=r(a, Q)$, and $K_{1}=r(y, A)$. Since $a=1+x=$ $(1-g)+y$, with $y \in g Q$, we have $K=r(y, Q) \cap g Q$. Hence $K_{1}=r(y, g Q g)=K \cap Q g=$ $K g$. By assumption $\mu(K)=\alpha$, hence $\mu(K)=\mu(g Q)$. Thus $Q K=Q g Q$ by Proposition 1 . Now

$$
\begin{aligned}
A K_{1} & =(g Q g)(K g)=g(Q K) g \quad \text { (because } g K=K) \\
& =g(Q g Q) g \quad \text { (from above) } \\
& =g Q g .
\end{aligned}
$$

Hence $A K_{1}=A$. Write $g=e+f$, where $e$ and $f$ are orthogonal idempotents of $A$ such that $y A=e A$. Then $a Q=(1-g) Q \oplus y Q=(1-g) Q \oplus e Q$ and $g Q=e Q \oplus f Q$, and therefore $f Q$ is a complement of $a Q$. By assumption $\mu(f Q)=\mu$-codim $(a Q)=\alpha$, giving $\mu(f Q)=$ $\mu(g Q)$. Hence $Q f Q=Q g Q$ by Proposition 1 . Now

$$
\begin{aligned}
A f A & =(g Q g) f(g Q g)=g(Q f Q) g=g(Q g Q) g \\
& =g Q g \\
& =A .
\end{aligned}
$$

Since $f A$ is a complement of $y A$ in $A$, this completes the verification of $\left(^{*}\right)$.

Observe that $g Q g$ is a prime, regular, right self-injective ring because $Q$ is such a ring. Also $g Q$ is directly infinite since $\mu(g Q)=\alpha>\kappa_{0}$. Thus $g Q g$ is a directly infinite ring. By Lemma 9, we have $y=g_{1} g_{2} \ldots g_{n}$ for some idempotents $g_{i} \in g Q g$. Hence

$$
\begin{aligned}
a & =(1-g)+y \\
& =\left[(1-g)+g_{1}\right]\left[(1-g)+g_{2}\right] \ldots\left[(1-g)+g_{n}\right]
\end{aligned}
$$

is a product of idempotents.

For the second part of the converse, assume (ii). Let $x=a-1$ and proceed exactly as above to produce $g=g^{2} \in H\left(\aleph_{0}\right)$ such that $x \in g Q g$. Let $y=g+x \in g Q g$. Since $g Q$ is directly finite, $g Q g$ is a directly finite, prime, regular, right self-injective ring. Also $y$ is a non-unit of $g Q g$ because $a=(1-g)+y$ is a non-unit of $Q$. By Corollary $4, y=g_{1} g_{2} \ldots g_{n}$ for some idempotents $g_{i} \in g Q g$ and the proof can now be completed exactly as above.

When $Q$ is directly finite (Type $\mathrm{I}_{\mathrm{f}}$ or $\mathrm{II}_{\mathrm{f}}$ ), $Q=H\left(\boldsymbol{\aleph}_{0}\right)$. The conditions in Theorem 6 then reduce to (ii), which is just the condition in Corollary 4 . At the other extreme, when $Q$ is Type III, $H\left(\aleph_{0}\right)=0$ and the conditions reduce to (i). If in addition $Q$ is simple, then this simply says that $a$ is neither left nor right invertible. Combining this with Corollary 4 we obtain the following further extension of Erdos' result [3] to arbitrary simple, right self-injective rings (whether directly finite or not). 
Corollary 11. Let $Q$ be any simple, right self-injective ring and let $a \in Q, a \neq 1$. Then $a$ is a product of idempotents if and only if $a$ is neither left nor right invertible.

In the Type I case, Theorem 6 provides the following corollary,

CoROLlary 12 (Reynolds and Sullivan [7]). Let $V$ be an arbitrary (right) vector space over a division ring $D$, and let $L(V)$ be the multiplicative semigroup of all linear transformations from $V$ into itself. Then an element $a \in L(V)$ can be written as a product of proper $(\neq 1)$ idempotents if and only if either

or

(1) $n(a)=d(a)=s(a) \geqslant \aleph_{0}$

(2) $0<n(a)=d(a) \leqslant s(a)<\aleph_{0}$,

where

$$
\begin{aligned}
& n(a)=\operatorname{dim} \operatorname{Ker}(a) \quad \text { (nullity of } a), \\
& d(a)=\operatorname{codim} \operatorname{Im}(a) \quad(\text { defect of } a), \\
& s(a)=\operatorname{codim}\{u \in V \mid a(u)=u\} \quad \text { (shift of } a \text { ). }
\end{aligned}
$$

Proof. Let $Q$ be the full linear ring $\operatorname{End}_{D}(V)$. As is well-known, $Q$ is a prime, regular, right self-injective ring (of Type I). See [4, Theorem 9.12]. We show that conditions (1) and (2) are respectively equivalent to conditions (i) and (ii) of Theorem 6.

Firstly observe that for $x, y \in Q$, we have $x Q \cong y Q$ (as right $Q$-modules) if and only'if $x V \cong y V$ (as vector spaces). Consequently, for $x \in Q$ and a cardinal $\alpha$,

Hence

$$
\alpha(x Q) \leqslant x Q \text { iff } \alpha(x V) \leqslant x V
$$

$$
\mu(x Q)=\left\{\begin{array}{lll}
0 & \text { if } & \operatorname{dim} x V=0, \\
\aleph_{0} & \text { if } 0<\operatorname{dim} x V<\kappa_{0}, \\
(\operatorname{dim} x V)^{+} & \text {if } \operatorname{dim} x V \geqslant \kappa_{0}
\end{array}\right.
$$

(where $\alpha^{+}$is the successor cardinal of $\alpha$ ).

Let $a \in Q$. Write $a=a b a$ for some $b \in Q$, and let $e=a b, f=b a$. Then $n(a)=$ $\operatorname{dim}(1-f) V, d(a)=\operatorname{dim}(1-e) V$, and $s(a)=\operatorname{dim}(1-a) V$. Hence

$$
\begin{aligned}
& n(a)=d(a)=s(a) \geqslant \aleph_{0} \\
\Leftrightarrow & n(a)^{+}=d(a)^{+}=s(a)^{+}>\aleph_{0} \\
\Leftrightarrow & \mu((1-f) Q)=\mu((1-e) Q)=\mu((1-a) Q)>\aleph_{0} \\
\Leftrightarrow & \mu(r(a, Q))=\mu \text {-codim }(a Q)=\mu((1-a) Q)>\aleph_{0} .
\end{aligned}
$$

Thus (1) and (i) are equivalent. Also

$$
\begin{aligned}
& 0<n(a)=d(a) \leqslant s(a)<\aleph_{0} \\
\Leftrightarrow & \operatorname{dim}(1-a) V<\aleph_{0} \text { and } a \text { is not a unit } \\
& \left(\text { since } n(a)=d(a) \text { always holds when } \operatorname{dim}(1-a) V<\aleph_{0}\right) \\
\Leftrightarrow & a=1+x, x \in \text { socle } Q, a \text { not a unit. }
\end{aligned}
$$

Now for a full linear ring, $H\left(\aleph_{0}\right)=$ socle $Q$, and so (2) is equivalent to (ii). 
We conclude by asking if characterizations similar to Theorems 3 and 6 hold in other regular rings, for example regular right self-injective rings in general (perhaps using general comparability [4, Theorem 9.14] in place of comparability, the more general infinite dimension functions $\mu_{M}$ in [4, Chapter 12] in place of $\mu$, and the relative dimension functions $d_{M}(-: R)$ in [4, Chapter 11] in place of a rank function) or $\aleph_{0}$-continuous regular rings (using [4, Theorems 14.33, 18.27]). In this vein, one obvious extension of Corollary 5 clearly does hold, viz:

An element $a$ of an Artinian regular ring $R$ is a product of idempotents if and only if for each maximal ideal $M$ of $R$, either $1-a \in M$ or $a+M$ is not a unit of $R / M$.

\section{REFERENCES}

1. R. J. H. Dawlings, Products of idempotents in the semigroup of singular endomorphisms of a finite-dimensional vector space, Proc. Roy. Soc. Edinburgh Sect. A 91 (1981), 123-133.

2. R. J. H. Dawlings, The idempotent generated subsemigroup of the semigroup of continuous endomorphisms of a separable Hilbert space, Proc. Roy. Soc. Edinburgh Sect. A 94 (1983), 351-360.

3. J. A. Erdos, On products of idempotent matrices, Glasgow Math. J. 8 (1967), 118-122.

4. K. R. Goodearl, Von Neumann regular rings (Pitman, 1979).

5. J. M. Howie, The subsemigroup generated by the idempotents of a full transformation semigroup, J. London Math. Soc. 41 (1966), 707-716.

6. K. C. O'Meara, Right orders in full linear rings, Ph.D. Thesis, University of Canterbury (1971).

7. M. A. Reynolds and R. P. Sullivan, Products of idempotent linear transformations, Proc. Roy. Soc. Edinburgh Sect. A 100 (1985), 123-138.

DePARTMENT OF MATHEMATICS

UNIVERSITY OF CANTERBURY

ChristChURCH

New Zealand 